\title{
A Rare Cause of Pneumoperitoneum: Perforated Gastrointestinal Stromal Tumor (GIST)
}

\author{
Thukirtha Manoharan ${ }^{1 *}$, Mahin Mohammadi ${ }^{2}$, Orhan Bulut ${ }^{1}$ \\ ${ }^{1}$ Department of Surgical Gastroenterology, Copenhagen University Hospital Hvidovre, Copenhagen, Denmark \\ ${ }^{2}$ Department of Pathology, Copenhagen University Hospital Hvidovre, Copenhagen, Denmark \\ Email: Thukirtha.Manoharan@regionh.dk
}

Received 3 April 2014; revised 7 May 2014; accepted 15 May 2014

Copyright (C) 2014 by authors and Scientific Research Publishing Inc.

This work is licensed under the Creative Commons Attribution International License (CC BY). http://creativecommons.org/licenses/by/4.0/

(c) () Open Access

\begin{abstract}
Gastrointestinal stromal tumor (GIST) is the most common mesenchymal tumor and has a malignant potential. The clinical presentation with pneumoperitoneum and peritonitis is extremely rare. We report a case of a 40-year-old male presented with symptoms of acute abdomen. Radiological work-up confirmed pneumoperitoneum. Emergency laparatomy and complete resection were performed. The final diagnosis revealed perforated GIST originating from the jejunum. If an abdominal mass presents with pneumoperitoneum and peritonitis, jejunal GIST should be considered in diagnosis. A complete radical resection followed by postoperative adjuvant chemotheraphy with Imatinib is recommended.
\end{abstract}

\section{Keywords}

Gastrointestinal Stromal Tumor, GIST, Pneumoperitoneum, Perforation, Small Intestine

\section{Introduction}

GISTs account for approximately $0,1 \%$ - 3\% of all gastrointestinal neoplasms and often present with vague symptoms [1]. About $60 \%$ of GISTs occur in the stomach, $20 \%-30 \%$ occur in the small intestine, and $10 \%$ occur in other parts of the GI tract. GISTs of the colon, rectum, and esophagus are rare [2]. The most commonly known symptoms of small bowel GISTs are abdominal pain (74\%), abdominal mass (72\%), GI-bleeding (44\%), partial or complete small bowel obstruction (44\%) [3]. The predominant cause of non-traumatic pneumoperitoneum is perforated viscus. $14 \%$ of all radiological confirmed pneumoperitoneum are due to malignancy [3].

\footnotetext{
${ }^{*}$ Corresponding author.
}

How to cite this paper: Manoharan, T., Mohammadi, M. and Bulut, O. (2014) A Rare Cause of Pneumoperitoneum: Perforated Gastrointestinal Stromal Tumor (GIST). Open Journal of Gastroenterology, 4, 237-241.

http://dx.doi.org/10.4236/ojgas.2014.45035 
Spontaneous perforation of GIST is an extremely rare presentation (0.8\%) [4]. Therefore, we present a rare case of a perforated GIST as a cause of pneumoperitoneum in a patient admitted with the symptoms of acute abdomen.

\section{Case}

A 40-year-old male was admitted to the emergency surgical unit with severe abdominal pain, fever and vomitings. He had no weight loss or blood in the stool, no prior history of admittance or co-morbidity. Physical examination showed signs of perforated bowel with peritoneal reaction in the left iliac fossa and high body temperature. Blood test with complete blood count revealed leukocytosis with a left shift, and elevated C-Reactive Protein (CRP) indicating infection. With the suspicion of acute diverticulitis, an abdominal computed tomography (CT) scan was preformed. The CT scan revealed pneumoperitoneum (Figure 1). Moreover, there was an accumulation of fluid, air and small bowel loops localised to the lower middle part of the abdomen. The radiological provisional diagnosis of perforated diverticulitis was made. Preoperative optimization with antibiotics and correction of electrolyte imbalance was initiated before the patient was taken to the operation room.

An emergency laparotomy revealed diffuse peritonitis with brownish fluid in the abdomen. A $13 \times 7 \times 5 \mathrm{~cm}$ anti-mesenteric tumor originating from the jejunum $45 \mathrm{~cm}$ distal to the ligament of Treizt was found (Figure 2). There was no sign of invasion of the tumor into adjacent organs or parietal peritoneum. The tumor was resected as en bloc with small intestine and a primary end-to-end, 2-layer anastomosis was performed. The postoperative period was uneventful. The patient was discharged on postoperative day 6 and he was referred to the department of oncology for adjuvant chemotheraphy.

Gross examination of the specimen revealed an outward bulging mass, which was centrally necrotic and contained hemorrhagic-necrotic material (Figure 3).

Histopathological assessment of the tumor showed a well delineated epithelioid gastrointestinal stromal tumor (Figure 4) originating from the small bowel wall. Tumor cells did not infiltrate the mucosa but only muscularis propria. Based on the size, location and the number of mitoses (5/50 HPFs), it was considered as a borderline malignancy.

KIT (CD117) showed strong diffuse cytoplasmic staining of tumor cells (Figure 5). Mutational analysis identified c-Kit mutation (p. W557R) and lacking mutation in PDGFRA genes.

There was no lymph node metastasis. It confirmed complete resection with clear surgical margins.

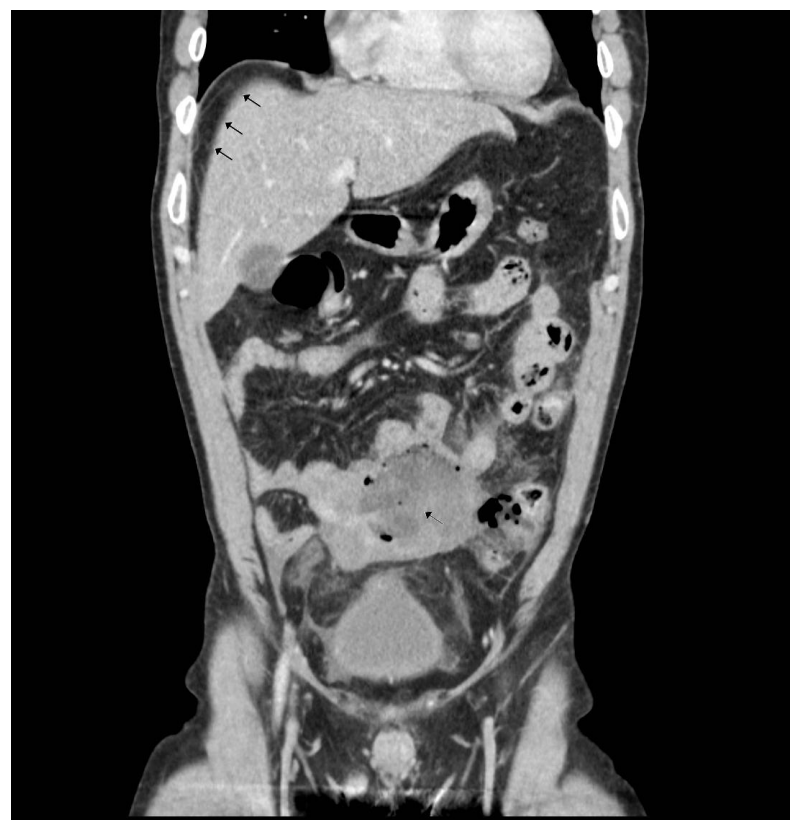

Figure 1. Coronal view of contrast-enhanced CT scan revealing sub-diaphragmatic free air (3 arrows subdiagphramatic) and an accumulation of fluid, air and bowel loops (1 arrow mid-adbominally). 


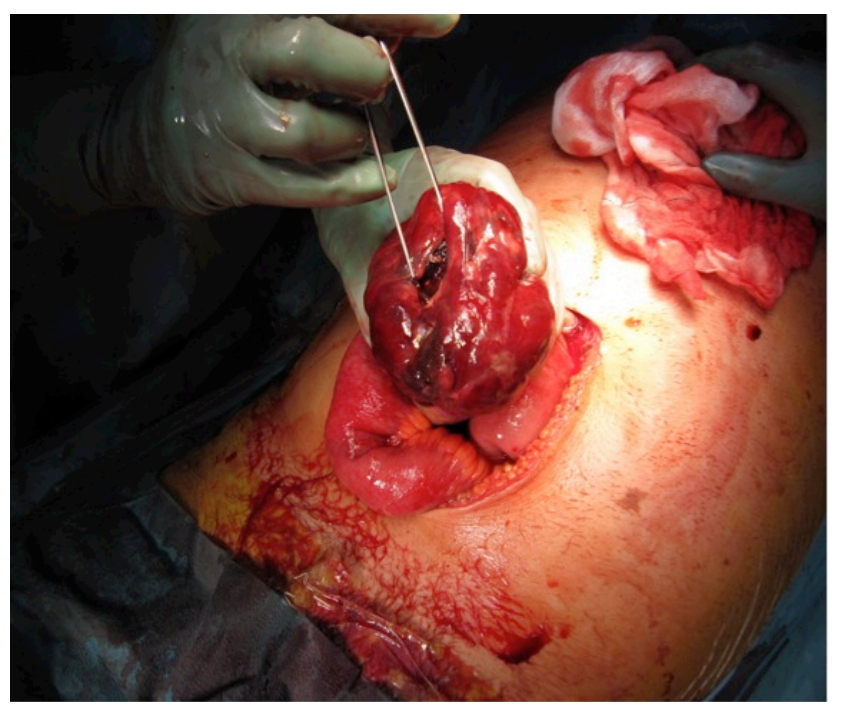

Figure 2. Intraoperative view of perforation site of tumor pointed by a pincette.

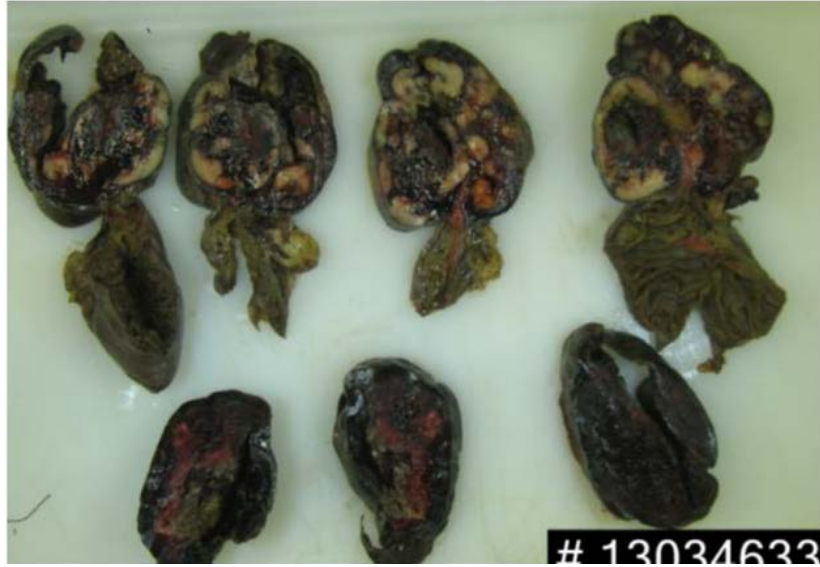

Figure 3. Gross appearance of the tumor illustrating intact overlying mucosa. The cut surface is granular and shows areas of hemorrhage and necrosis.

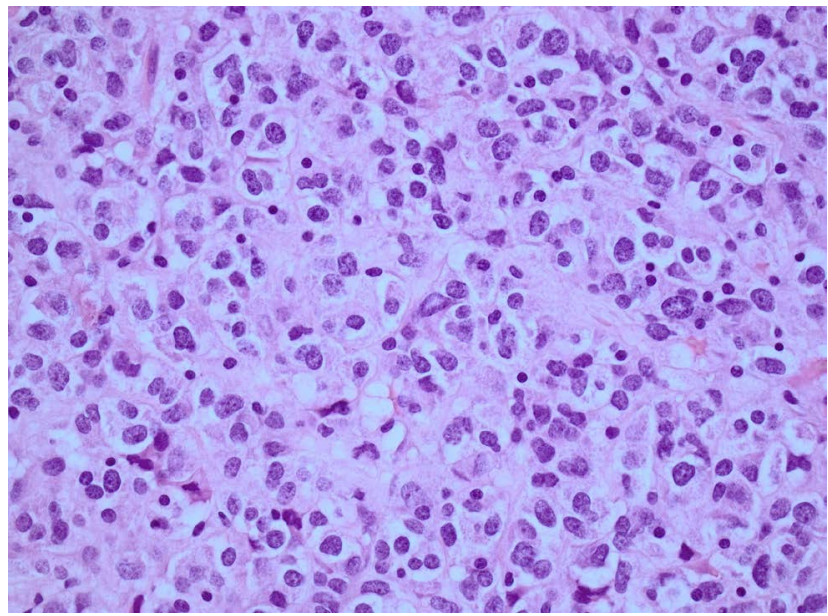

Figure 4. Epithelioid cell GIST stained by hematoxylin eosin $(\times 400)$. 


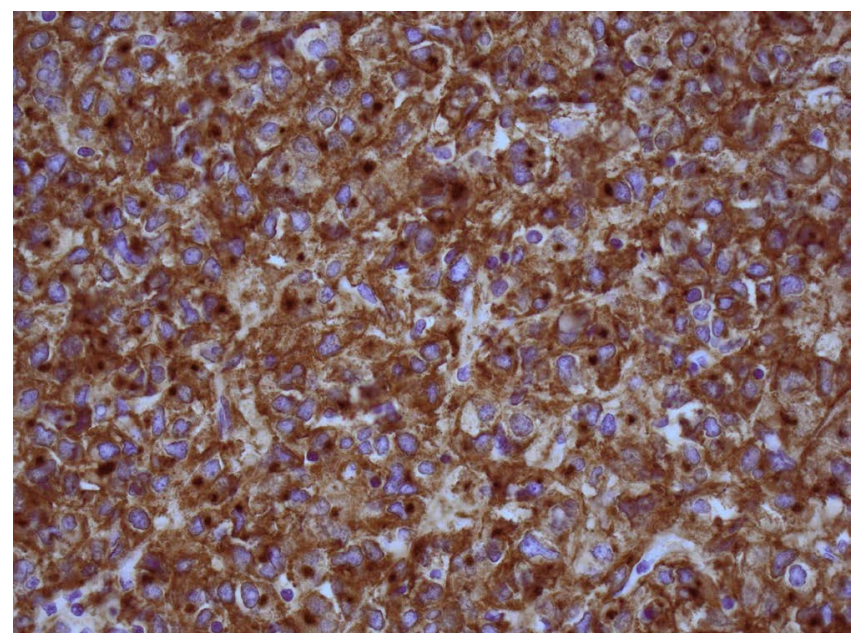

Figure 5. Strong diffuse cytoplasmic positivity for KIT (CD117) (IHC, ×400).

\section{Discussion}

GIST affects mainly adults over 40 years (90\%) [1]. Diagnosis is often delayed until complications develop such as bleeding, obstruction or perforation.

GIST was first described by Mazur and Clark (1983) [5]. Since then there have been 15 cases reported on perforation of jejunal GIST [6]. There is only one case report recently reported on perforation of stomach GIST [7]. All reported jejunal GIST perforations in the literature showed that the patients were admitted with severe abdominal pain and signs of pneumoperitoneum, which in general is not specific for GIST [6]-[10]. However, in one of the reported cases, the patient had two episodes of upper gastrointestinal hemorrhage treated conservatively two years prior to admittance. Another study suggested that there was a correlation between small bowel tumors and clinical emergency presentation [11]. The most commen presentation for GIST is though bleeding [10] [11]. Perforation is unique and extremely rare [6] [8]-[10]. Therefore when patients are admitted with signs of pneumoperitoneum, the provisional diagnosis is often perforated upper gastrointestinal ulcer or diverticulitis. CT scans can in these cases be a useful diagnostic tool. In our case, an acute diverticulitis was suspected preoperatively due to the patient's pain in the left iliac fossa, high body temperature, leukocytosis and elevated CRP.

The nonspecific and variable clinical symptom of a jejunal GIST is a diagnostic challenge. CT scans are the most commonly used diagnostic modality and offer two advantages. One advantage is to rule out other possible causes of pneumoperitoneum and the other is to determine the location of the primary tumor, as well as any radiological signs of metastasis. This will help the surgeon preoperatively to choose the correct surgical strategy: Whether it is to make a complete resection of tumor (no signs of malignancy) or to perform a palliative procedure i.e., intestinal bypass or stoma. Approximately $20 \%$ - 30\% of all GISTs are malignant at the time of initial diagnosis with the criteria of metastasis and adjacent organs invasion [4].

The pathology of GIST is due to a gain-in-function mutation. Sornmayura reported that GISTs with epithelioid morphology are linked with a malignant course [4]. Moreover another study confirmed that the overall survival of the patients with spindle cell tumors was significantly longer than epithelioid tumors; and epithelioid cell type was significantly associated with poor outcome [12]. The most consistent histopathological features used to predict aggressiveness are tumor size and mitotic index. Tumors with a mitotic rate of $>5 / 50$ high-power field usually has a malignant behavior. Even though the mitotic index was 5 in our case, the patient was in the borderline risk group due to the size of tumor and the epithelioid morphology in the histopathological examination.

A complete surgical resection of the tumor is the only potentially curative treatment for the patients, who have primary, resectable GIST. A systematic lymphadenectomy is not necessary due to low risk of lymph node metastases [13]. Microscopic assessment needs to be done to determine the origin of tumor and to classify the tumor in low or high risk category tumors. Postoperative Imatinib treatment is needed for optimal treatment [6] [9] [10]. The assessment of relapse risk for GIST patients is based on the mitotic rate, tumor size and site, surgical margins and the status of tumor rupture. The five-year survival in cases of small intestinal GIST perforation was 
reported as $24 \%$ [1].

\section{Conclusion}

In conclusion, we present a case of perforated jejunal GIST causing pneumoperitoneum. Tumor presentation with perforation and peritonitis has one of the worst clinical outcomes; therefore a preoperative workup with CT scan is essential in diagnosis and planning of the surgical strategy. So if an abdominal mass presents with pneumoperitoneum and peritonitis, a small intestinal GIST should be considered. A complete surgical resection of the mass with free margin is necessary for radical treatment.

\section{Disclosures}

The authors declare that they have no competing interests.

\section{References}

[1] Crosby, A., Catton, C.N., Davis, A., Couture, J., O’Sullivan, B., Kandel, R. and Swallow, C.J. (2001) Malignant Gastrointestinal Tumors of the Small Intestine: A Review of 50 Cases from a Prospective Database. Annals of Surgical Oncology, 8, 50-59. http://dx.doi.org/10.1007/s10434-001-0050-4

[2] Miettinen, M., El-Rifai, W., HL Sobin, L. and Lasota J. (2002) Evaluation of Malignancy and Prognosis of Gastrointestinal Stromal Tumors: A Review. Human Pathology, 33, 478-483. http://dx.doi.org/10.1053/hupa.2002.124123

[3] Kumar, A., et al. (2012) The Etiology of Pneumoperitoneum in the 21st Century. Journal of Trauma and Acute Care Surgery, 73, 542-548.

[4] Sornmayura, P. (2009) Gastrointestinal Stromal Tumors (GISTs): A Pathology View Point. Journal of the Medical Association of Thailand, 92, 124-135.

[5] Mazur, M.T. and Clark, H.B. (1983) Gastric Stromal Tumors. Reappraisal of Histogenesis. American Journal of Surgical Pathology, 7, 507-519. http://dx.doi.org/10.1097/00000478-198309000-00001

[6] Roy, S.D., Khan, D., De., K.K. and De., U. (2012) Spontaneous Perforation of Jejunal Gastrointestinal Stromal Tumor. Case Report and Review of Literature. World Journal of Emergency Surgery, 7, 37. http://dx.doi.org/10.1186/1749-7922-7-37

[7] Skipworth, J.R., Fanshawe, A.E., West, M.J. and Al-Bahrani, A. (2014) Perforation as a Rare Presentation of Gastric Gastrointestinal Stromal Tumors: A Case Report and Review of the Literature. Annals of The Royal College of Surgeons of England, 96, 96E-100E.

[8] Sjogren, P.P., Banerji, N., Batts, K.P., Graczyk, M.J. and Dunn, D.H. (2013) Rare Presentation of Gastrointestinal Stromal Tumor with Spontaneous Esophageal Perforation: A Case Report. International Journal of Surgery Case Reports, 4, 636-639. http://dx.doi.org/10.1016/j.ijscr.2013.02.029

[9] Memmi, N., Cipe, G., Bektasoglu, H., Toydemir, T., Kadioglu, H., Bozkurt, S., Buyukpinarbasili, N., Karatepe, O. and Muslumanoglu, M. (2012) Perforated Gastrointestinal Stromal Tumor in the Jejunum: A Rare Cause of Acute Abdomen. Oncology Letters, 4, 1244-1246.

[10] Efremidou, E.I., Liratzopoulos, N., Papageorgiou, M.S. and Romanidis, K. (2006) Perforated GIST of the Small Intestine as a Rare Cause of Acute Abdomen: Surgical Treatment and Adjuvant Therapy. Case Report. Journal of Gastrointestinal and Liver Diseases, 15, 297-299.

[11] Catena, F., Ansaloni, L., Gazzotti, F., Gagliardi, S., Di Saverio, S., De Cataldis, A. and Taffurelli, M. (2005) Small Bowel Tumors in Emergency Surgery: Specificity of Clinical Presentation. ANZ Journal of Surgery, 75, 997-999. http://dx.doi.org/10.1111/j.1445-2197.2005.03590.x

[12] Demir, L., Ekinci, N., Erten, C., Kucukzeybek, Y., Alacacioglu, A., Somali, I., Can, A., Dirican, A., Bayoglu, V., Akyol, M., Cakalagaoglu, F. and Tarhan, M.O. (2013) Does Immunohistochemistry Provide Additional Prognostic Data in Gastrointestinal Stromal Tumors? Asian Pacific Journal of Cancer Prevention, 14, 4751-4758.

[13] Fong, Y.M., Coit, D.G., Woodruff, J.M. and Brennan, M.F. (1993) Lymph Node Metastasis from Soft Tissue Sarcoma in Adults. Analysis of Data from a Prospective Database of 1772 Sarcoma Patients. Annals of Surgery, 217, 72-77. http://dx.doi.org/10.1097/00000658-199301000-00012 